\title{
Review \\ Gamma-Ray Bursts at TeV Energies: Observational Status
}

\author{
Koji Noda ${ }^{1, *,+}$ (D) and Robert Daniel Parsons ${ }^{2, *,+}+\mathbb{D}$ \\ 1 Institute for Cosmic Ray Research, The University of Tokyo, 5-1-5 Kashiwanoha, Kashiwa 277-8582, Japan \\ 2 Institut für Physik, Humboldt-Universität zu Berlin, Newtonstr. 15, D-12489 Berlin, Germany \\ * Correspondence: nodak@icrr.u-tokyo.ac.jp (K.N.); daniel.parsons@physik.hu-berlin.de (R.D.P.) \\ + These authors contributed equally to this work.
}

check for

updates

Citation: Noda, K.; Parsons, R.D. Gamma-Ray Bursts at TeV Energies: Observational Status. Galaxies 2022 10, 7. https://doi.org/10.3390/ galaxies10010007

Academic Editor: Junhui Fan

Received: 9 December 2021 Accepted: 25 December 2021 Published: 5 January 2022

Publisher's Note: MDPI stays neutral with regard to jurisdictional claims in published maps and institutional affiliations.

Copyright: (C) 2022 by the authors Licensee MDPI, Basel, Switzerland. This article is an open access article distributed under the terms and conditions of the Creative Commons Attribution (CC BY) license (https:// creativecommons.org/licenses/by/ $4.0 /)$.

\begin{abstract}
Gamma-ray bursts (GRBs) are some of the most energetic events in the Universe and are potential sites of cosmic ray acceleration up to the highest energies. GRBs have therefore been a target of interest for very high energy gamma-ray observatories for many years, leading to the recent discovery of a number of bursts with photons reaching energies above $100 \mathrm{GeV}$. We summarize the GRB observational campaigns of the current generation of very high energy gamma-ray observatories as well as describing the observations and properties of the GRBs discovered so far. We compare the properties of the very high energy bursts to the total GRB distribution and make predictions for the next generation of very high energy gamma-ray observations.
\end{abstract}

Keywords: gamma-ray bursts; VHE gamma-rays; IACTs

\section{Introduction}

The origins of gamma-ray bursts have been a mystery since their discovery in the 1960s. As the name suggests, the definition was an unknown phenomenon, rather than an astronomical object, a short pulse seen in gamma-ray bands $(>\sim 100 \mathrm{keV})$ detected by the Vela gamma-ray detection satellites [1]. After this early detection, tremendous efforts were devoted to this research field for several decades. We have come to know that GRBs are extra-Galactic phenomena, occurring isotropically over the sky at any time, with a variety of properties in time and energy. In addition, it became clear that there are multiple classes in this object, whose origins are different. Either class of bursts has a short pulse of gamma-/X-rays called "prompt" emission, followed by a quickly fading "afterglow" detected in a wider waveband (See Granot and Gill in this special issue for more details on GRB physics).

Deeper insights into the origin and phenomenology of GRBs were made by dedicated detectors placed on satellites, such as BATSE [2] on board the Compton gamma-ray observatory (CGRO) and HETE-2 [3]. These instruments confirmed the extragalactic origin of the bursts [4], a clearly bimodal distribution in their emission timescales [5] and a characteristic spectral shape [6]. The Neil Gehrels Swift Observatory (Swift [7]) was launched in 2004 and made a big step toward the full understanding of GRBs. The Swift observatory has three instruments named the Burst Alert Telescope (BAT), X-ray Telescope (XRT) and UltraViolet/Optical Telescope (UVOT). The Swift-BAT is sensitive in 15-150 keV energies, and has the ability to localize the direction of the incoming gamma rays, typically with an error of a few arcmin. This small localization error enables other telescopes on the Earth and onboard to repoint and observe the burst without any ambiguity. In order to organize a quick response all over the world, a network called Gamma-ray Coordination Network (GCN) system is established [8]. The GCN sends to subscribed astronomers a series of packets and emails containing the coordinates and other properties of the detected GRBs. The typical delay from the onset of the burst to the arrival of information is short, typically taking around $20 \mathrm{~s}$. The bunch of information is called "alert", meant to trigger a quick response ("follow-up") of those following the alert system. A large part of the success in the Swift era can be attributed to such follow-up observations by the optical/infrared/radio 
telescopes. The studies revealed a big picture, such as the mechanism of the afterglow and properties of typical host galaxies, and confirmed the classification by the burst duration that was suggested by BATSE. Properties of the afterglow such as flares and plateau phase found in X-rays remained a mystery.

There are other satellites that contribute to sending their GRB alerts. Among them, one of the most active satellites is the Fermi Gamma-ray Space Telescope (Fermi) launched in 2008. It has two instruments called Gamma-ray Burst Monitor (GBM [9]) and Large Area Telescope (LAT [10]). The Fermi-GBM is sensitive from a few $\mathrm{keV}$ to $\sim \mathrm{MeV}$, and has a $4 \pi$ field of view with a localization error of up to 15 degrees. The Fermi-GBM increased the number of detected GRBs roughly to once every 2 days (from once every 5-7 days in the Swift era), and enabled statistical studies of thousands of GRBs [11]. However, the remaining uncertainties such as the mechanism of the prompt emission, and the origin of the central engine, have been a mystery even in the Fermi era. Indeed, the increase of the number of GRBs revealed further unknowns, in particular, in the GRB classification (e.g., [12]).

The Fermi-LAT is a pair conversion telescope sensitive to $\mathrm{MeV}-\mathrm{GeV}$ gamma-rays, with a field of view of around 0.5 steradians. The Fermi-LAT confirmed the existence of GeV-emitting GRBs [13] that were suggested by the predecessor EGRET telescope, and investigated their emission properties in detail. It has been found that the $\mathrm{GeV}$ gamma-ray emission is often observed with a delay of (typically) about 1-10 s after the burst onset. For a few $\mathrm{GeV}$ GRBs, gamma rays with energy of tens of $\mathrm{GeV}$ were detected even hours after the burst, which challenges the standard model of the relativistic particle acceleration. To push beyond the $100 \mathrm{GeV}$ energy limit, a satellite instrument such as Fermi has difficulty detecting gamma rays due to its limited collection area (about $1 \mathrm{~m}^{2}$ ). These energies are instead within the reach of imaging atmospheric Cherenkov telescopes (IACTs), which have an orders of magnitude larger effective detection area. However, it should be noted that an ultimate high energy limit is placed on the detection of distant photons due to the absorption of photons by the predominantly infra-red extragalactic background light (EBL) [14]. EBL absorption becomes more important as both energy and source distance increase, causing an attenuation of energetic photons to have a steepening effect on the source spectrum. Therefore, it has long been expected that IACTs could detect nearby and bright GRBs and contribute to further understanding the GRB phenomena.

\section{Observation Schemes}

The current generation of IACTs has been hugely successful in transforming the field of VHE gamma-ray astronomy from a field with only a handful of sources detected to over 200 [15]. Significant effort has been invested over several generations of IACT instruments to perform rapid transient source follow ups with, optimising for high sensitivity to steep spectrum sources. A major focus for transient observations in these instruments is placed on GRBs, in parallel to very similar observation programs for related sources such as gravitational wave and VHE neutrino triggers. The sensitivity of the three current instruments is roughly similar, being around three to four orders of magnitude better than that of the Fermi-LAT for an observation time shorter than $10^{4} \mathrm{~s}$ [16].

\subsection{H.E.S.S.}

The High Energy Stereoscopic System (H.E.S.S.) is an array of five imaging atmospheric Cherenkov telescopes located in the Khomas highlands of Namibia (approx. $23.5^{\circ}$ latitude) at an altitude of $1800 \mathrm{~m}$. The H.E.S.S. array is composed of four $12 \mathrm{~m}$ diameter telescopes (approx. $100 \mathrm{~m}^{2}$ mirror area) in a square of $12 \mathrm{~m}$ side length with a $28 \mathrm{~m}$ telescope (approx. $600 \mathrm{~m}^{2}$ mirror area) at the array center with an energy threshold as low as $40 \mathrm{GeV}$. The differential sensitivity of the system is estimated below $1 \%$ of the Crab Nebula flux above $300 \mathrm{GeV}$ with a 50-h observation [17].

H.E.S.S. has maintained an active GRB observation campaign since its commissioning in 2004 [18], which received renewed focus in 2012 [19] with the completion of the large telescope (and subsequent lowering of the energy threshold). Trigger and observability 
criteria have been continuously adapted and improved over the lifetime of H.E.S.S. based on the experience of observations and the instruments available to trigger observations. The current observation scheme automatically monitors events from the GCN system and classifies them into two categories [20]. The first category being events immediately visible from the H.E.S.S. site at the time of the burst, or within $4 \mathrm{~h}$, which are only subject to observability requirements, with any event visible above $60^{\circ}$ zenith angle for more than $30 \mathrm{~min}$ being observed. Observation of these events is initiated automatically with no human input. The remaining bursts are subjected to requirements based on the source significance (specifically in the case of Fermi-GBM observations) and redshift (if known), with longer observation delays being allowed for nearer objects. However, any observation restrictions can be overridden by burst advocates in the case of confirmed high energy gamma-ray emission from the Fermi-LAT.

In the period from 2004 to 2019, H.E.S.S. observed a total of 96 GRBs [18,21] split between four telescope (32 observations) and five telescope data (64 observations).

\section{2. $M A G I C$}

MAGIC (Major Atmospheric Gamma Imaging Cherenkov telescopes) is a system composed of two imaging air Cherenkov telescopes, located at $2200 \mathrm{~m}$ above sea level, at the Roque de Los Muchachos Observatory in La Palma, Canary Islands, Spain. Both telescopes have a mirror diameter of $17 \mathrm{~m}$, which enables a low energy threshold of above $30 \mathrm{GeV}$, depending on the observing mode and conditions, with a field of view of $\sim 10$ square degrees. The integral sensitivity of the system is $0.66 \%$ of the Crab Nebula flux above $220 \mathrm{GeV}$ with a 50-h observation [22].

The telescopes were designed to perform fast follow-up observations of GRBs. Their structure is made of light-weight carbon-fiber-reinforced polymer tubes, and their drive system has a special fast slewing mode with a speed of $7 \mathrm{deg} / \mathrm{s}$. To reduce the latency in performing GRB observations, MAGIC implemented a fully automatic reaction to alerts through its automatic alert system (AAS). AAS is a multi-threaded program that receives GCN notices containing the sky coordinates, filters them with predefined conditions, and sends commands to the central control software of the telescopes when needed. The AAS also includes a check of new targets according to a predefined priority. If multiple alerts are received for a single GRB, AAS selects the one with the best localization. Once the commands are sent to the central control software, it issues the following commands to the telescope subsystems such as drive, camera control, and DAQ, in order to slew and start to observe the GRB as early as possible.

The predefined conditions to trigger observations are the zenith angle less than 60 degrees, Moon distance larger than 30 degrees, and Sun distance larger than 103 degrees. The observation up to $4 \mathrm{~h}$ after $T_{0}$ is triggered automatically if the above conditions are met (Note that this criteria is updated after 2019 to allow more late time observations). In the period from 2005 (from 2013 after the major upgrade) to 2019, MAGIC observed a total of 107 (50) GRBs [23].

\subsection{VERITAS}

VERITAS [24] consists of an array of four 12-m imaging atmospheric Cherenkov telescopes located at the Fred Lawrence Whipple Observatory in southern Arizona, USA, at $1300 \mathrm{~m}$ above sea level. Each of the telescopes is equipped with a camera of PMTs covering a field of view (FoV) of 3.5 degrees. The system has maximum sensitivity in the energy range of $100 \mathrm{GeV}$ to $30 \mathrm{TeV}$. The angular resolution is $\sim 0.1$ degrees at $1 \mathrm{TeV}$ and the energy resolution is $15-25 \%$ at $1 \mathrm{TeV}$. The current system can detect a source ( $5 \sigma$ significance) with $1 \%$ of the Crab Nebula flux in $25 \mathrm{~h}$ [25].

VERITAS is subscribed to the GCN and has integrated an automated tracking response from incoming GCN alerts. The alerts are quickly parsed and reviewed by onsite operators, who assess visibility and observable conditions (such as pointing direction, slew time, and weather) and decide to follow up the alert. 
VERITAS has followed up more than 200 GRBs since the beginning of its GRB program in 2006 [26].

\subsection{Other Instruments}

This review concentrates primarily on the VHE GRB detections made by the current generation of IACTs (and the prospects for the future Cherenkov telescope array [27]). However, a new generation of sensitive wide field of view (wFOV) ground-based gammaray telescopes maintain strong GRB observation programs. wFOV gamma-ray instruments typically use ground based particle detectors (e.g., HAWC [28] \& Tibet-AS $\gamma$ [29]), sometimes in combination with wide field Cherenkov light detectors (e.g., LHAASO [30]). This detection technique typically has a higher energy threshold and reduced sensitivity in comparison to IACTs. However, the large field of view ( $\sim 2$ steradian) of these detectors make them much more likely to catch the GRB during its prompt phase. Eventually, these detectors might even provide triggers to IACTs on top of those from satellites. This detector class therefore has strong potential to extend the number of detected GRBs beyond that seen by the IACTs, potentially into time frames which are difficult or even impossible to access with Cherenkov telescopes.

\section{Status of Detected GRBs}

The observation programs of the current generation of VHE gamma-ray observatories in the previous sections have been performed for many years and have been able to provide useful upper limits and constraints on the VHE gamma-ray emission of GRBs [18,31-36]. However, in the last few years, several GRB detections have been made around $100 \mathrm{GeV}$. In this section, we will describe the observations of these detected events and the major findings from their analysis.

\subsection{GRB 180720B}

GRB 180720B was first detected by the Swift-BAT on 20 July 2018, 14:34:56 UT ( $\left.T_{0}\right)$ [37]. This GRB was relatively bright in X-ray emission in the prompt phase and long with a characteristic timescale of $90 \%$ of gamma-ray emission $\left(\mathrm{T}_{90}\right)$ of $\sim 49 \mathrm{~s}$ [38], classifying it as a long GRB. In the following hours, the fading of the prompt emission revealed an extremely bright X-ray afterglow [38], second only in brightness to the extreme GRB 130427A. A detection of the GRB by the Fermi-LAT and GBM followed around $10 \mathrm{~h}$ later, with a maximum photon energy of $5 \mathrm{GeV}$ being detected [39]. Numerous optical observations of the afterglow resulted in the measurement of a redshift by the VLT/X-Shooter of 0.654 [40] around $19 \mathrm{~h}$ after burst time.

At the time of the GRB prompt emission, GRB 180720B was not visible from the H.E.S.S. site, only becoming observable above the typical 45 degree zenith angle threshold used by H.E.S.S. until $\mathrm{T}_{0}+10.1 \mathrm{~h}$ [41]. In total, around two hours of observations were made before observations were ended. When a full mono analysis of these data was later performed, a clear signal at 5.3 $\sigma$ was seen at a position consistent with the location of the GRB. By this time, the GRB observations from the H.E.S.S. site were no longer possible and follow-up observations were made at $T_{0}+18$ days, with no source being seen at this position.

The observed spectrum extracted in the energy range $100 \mathrm{GeV}$ to $440 \mathrm{GeV}$ was fit with a power law and found to be extremely steep, with a spectral index of -3.7 , explaining why the event was seen only in the low energy optimized mono analysis. After correcting the data for the absorption of energetic photons by the extragalactic background light [14], a rather hard spectral index of $-1.6 \pm 1.2$ was extracted. This hard index is consistent with the value of -2 seen in both $X$-ray and gamma-ray data, but with a rather large uncertainty due to the relatively narrow energy range in which the spectrum could be fit.

\subsection{GRB $190114 C$}

GRB 190114C was identified by Swift-BAT and Fermi-GBM on 14 January 2019, 20:57:03 UT $\left(T_{0}\right)$. Its duration in terms of $T_{90}$ was $\sim 116 \mathrm{~s}$ by Fermi-GBM [42] and $\sim 362 \mathrm{~s}$ by Swift-BAT [43], both of which indicate that it can be classified as a long GRB. Its afterglow 
emission is reported by various instruments from $1.3 \mathrm{GHz}$ to $23 \mathrm{GeV}$, and its redshift is reported to be $z=0.4245 \pm 0.0005[44,45]$. The isotropic-equivalent energy of the emission at energy of $1-10^{4} \mathrm{keV}$ was $E_{\mathrm{iso}} \approx 3 \times 10^{53} \mathrm{erg}$ by Fermi-GBM. The GRB was more energetic then the average but not exceptional.

The observation by MAGIC started from $T_{0}+57 \mathrm{~s}$ until $T_{0}+15,912 \mathrm{~s}$ in good weather but with a bright moonlight and high zenith angle over 55 degrees. Nevertheless, the detection above $0.2 \mathrm{TeV}$ in the first $20 \mathrm{~min}$ was reported by MAGIC through GCN [46] and ATel [47], which was just $4 \mathrm{~h}$ after the burst based on the result of a quick online analysis. It was the first announcement of a GRB detection with IACTs ever. Fermi-LAT observations confirmed that the detected emission is from the afterglow phase of the GRB, even at $T_{0}+57 \mathrm{~s}$ [48]. The light curve obtained by MAGIC is decaying monotonically mostly following the X-ray observations. It suggested that the amount of energy conveyed by VHE gamma rays is close to one in X-rays. The obtained spectrum shows a single power-law with a slope of index -2.2 after the spectrum corrected for EBL absorption, extending even to multi-TeV energies [49]. The multi-wavelength (MWL) study with 23 instruments showed that the MWL spectrum can be well modeled with the synchrotron self-Compton (SSC) emission, and that the VHE gamma-ray emissions is consistent with the estimation with SSC [50].

\subsection{GRB 190829A}

GRB 190829A was initially detected by the Fermi-GBM on 29 August 2019 at 19:55:53 UT [51] and was classified as a long GRB $\left(T_{90}=63 \mathrm{~s}\right)$, soon after being detected by the Swift-BAT and XRT $[52,53]$. The X-ray light curve showed an interesting structure with multiple re-brightening events, most notably significantly increasing in brightness around $1000 \mathrm{~s}$ after burst time. Due to the bright nature of this burst, multiple ground based optical observations were made, allowing the identification of the GRB host galaxy [54] and ultimately measuring the GRB redshift at a relatively very local $z=0.0785 \pm 0.0005$. Although this GRB was very bright, its brightness was largely due to its relative closeness and in fact was of rather low luminosity and had an isotropic energy release of only around $2 \times 10^{50} \mathrm{erg}$.

Observations with the H.E.S.S. array began $4 \mathrm{~h}$ after the burst, with a significant signal being detected for three consecutive observation nights (until $56 \mathrm{~h}$ after burst time) [55]. The intrinsic source spectrum was extracted using data from the four $12 \mathrm{~m}$ telescopes, with a best fit power law index of $-2.06 \pm 0.1$ on night one (in the energy range from $180 \mathrm{GeV}$ to $3.3 \mathrm{TeV}$ ) and $-1.86 \pm 0.26$ on night two, the significance of night 3 observations was too low to extract a reliable spectrum. Again, the recovered index was consistent with that seen in X-ray measurements both in index and flux level. The gamma-ray energy flux over the course of the observations was seen to decrease with a time decay index of $1.09 \pm 0.05$, consistent with the X-ray temporal decay.

Modeling of this GRB by the H.E.S.S. collaboration shows difficulties with reproducing the source spectrum using the conventional one zone SSC model [55]. Instead, they propose a synchrotron emission model; however, one must explain here the extension of the emission beyond the typical maximum synchrotron energy.

\subsection{GRB 201216C}

GRB 201216C is a long GRB triggered by the Swift-BAT on 16 December 2020, 23:07:31 $\mathrm{UT}\left(T_{0}\right)$ [56]. Its prompt emission with multiple peaks lasted for about $48 \mathrm{~s}$ in terms of $T_{90}$ measured by Swift-BAT [57], and 29.9 s by Fermi-GBM [58], both of which clearly classified it as a long GRB. Its redshift is estimated to be $z=1.1$ by the optical observations with the ESO Very Large Telescope [59]. The isotropic-equivalent energy of the emission at energy of $10-10^{3} \mathrm{keV}$ was $E_{\text {iso }} \approx 4.7 \times 10^{53} \mathrm{erg}$ with the fluence measured by Fermi-GBM.

MAGIC started to observe it at $T_{0}+56 \mathrm{~s}$, until $2.2 \mathrm{~h}$ after the burst. The zenith angle of the observation ranged from 37 to $68 \mathrm{deg}$, in good weather on a moonless night, which made possible the analysis of the data with an energy threshold below $100 \mathrm{GeV}$ (changing w.r.t the zenith angle). MAGIC detected the gamma rays with a significance of 5.9 sigma 
from the first $20 \mathrm{~min}$ of the observation [60,61]. The light curve obtained from the first $20 \mathrm{~min}$ (with an energy threshold of $70 \mathrm{GeV}$ ) was monotonically decaying, and the spectrum from the first $20 \mathrm{~min}$ is consistent with a power-law extended from $50 \mathrm{GeV}$ to $200 \mathrm{GeV}$ [61].

\section{Sub Threshold GRB Detections}

In addition to the GRBs passing the typical $5 \sigma$ significance threshold, there are several bursts which show lower significance hints of emission. Although these bursts should be treated with caution, they may contain hints of the physics of VHE gamma-ray emission in some classes of GRBs.

\subsection{GRB 160821B}

GRB 160821B is a short GRB triggered by the Swift-BAT on 21 August 2016, 22:29:13 UT $\left(T_{0}\right)$. Its prompt emission was a short-decaying pulse with a duration of $0.48 \mathrm{~s}$ in terms of $T_{90}$ measured by Swift-BAT [62], while about $1 \mathrm{~s}$ by Fermi-GBM [63]. A host galaxy is identified at redshift $z=0.16$ by the Nordic Optical Telescope [64], which made it one of the nearest short GRBs. The isotropic-equivalent energy of the emission at energy of $10-10^{3} \mathrm{keV}$ (measured by GBM [63]) was $E_{\mathrm{iso}} \approx 1.2 \times 10^{49} \mathrm{erg}$.

MAGIC started to observe it $24 \mathrm{~s}$ after its onset, with the zenith angle of $34 \mathrm{deg}$ up to 55 deg until $4 \mathrm{~h}$ after $T_{0}$. The observations were carried out under a strong moon light and adverse weather, which made the energy threshold higher than the typical value. Nevertheless, a hint of gamma-ray emission is obtained with a significance of 2.9 sigma (post-trial) above $0.5 \mathrm{TeV}$ [65]. The energy flux was estimated in a range of $0.5-5 \mathrm{TeV}$ and compared with a detailed multi-wavelength model. The emission from radio to X-ray bands are modeled with the forward shock, the reverse shock, and also the kilonova emission that was reported later in 2019 [66,67]. Based on the physics parameters obtained in the above modeling, the flux in the $\mathrm{TeV}$ band was estimated with a Synchrotron self-Compton model. However, the putative flux by MAGIC is about an order of magnitude higher than the estimation by the model, which suggests a possibility of other explanations for the $\mathrm{TeV}$ emission, such as the external Compton scattering [65].

\subsection{GRB 201015A}

GRB 201015A was detected by the Swift-BAT on 15 October 2020, 22:50:13 UT ( $\left.T_{0}\right)$ [68]. The duration of the prompt emission in terms of $T_{90}$ is $9.8 \mathrm{~s}$ in the $15-350 \mathrm{keV}$ band, from which the classification was not clear yet due to a complex light curve with a short pulse with a longer tail. The fluence in the $15-150 \mathrm{keV}$ band is $2.0 \times 10^{-7} \mathrm{erg} / \mathrm{cm}^{2}$ [69]. The redshift of the burst was reported by GTC to be $z=0.426$ [70], later confirmed by the NOT to be $z=0.423$ [71]. The isotropic equivalent prompt emission energy in the Fermi-GBM range is $E_{\mathrm{iso}} \approx 1.1 \times 10^{50} \mathrm{erg}$ [72]. Finally, a supernova component was identified in late optical observations, which confirmed its classification as a long GRB [73,74]

MAGIC started to observe it $33 \mathrm{~s}$ after the burst onset, which is one of the fastest among the GRB observations by MAGIC. It continued until $4 \mathrm{~h}$ later, with the zenith angle starting from 24 degrees to 48 degrees. Quick offline analysis showed a hint of gamma-ray signals from the GRB with a significance of $>3 \sigma$, as reported in GCN [75]. Final offline analysis extracted with a putative flux $(>140 \mathrm{GeV})$ is $0.7-4.0 \times 10^{-11} / \mathrm{s} / \mathrm{cm}^{2}$. Since its redshift is close to one of GRB 190114C, we can roughly expect its VHE gamma-ray flux from their $E_{\text {iso }}$ values. GRB 201015A has $E_{\text {iso }} 3$ orders of magnitude less than GRB 190114C, which is consistent with the flux, which is 2.5 orders of magnitude less, roughly scaling with the $E_{\text {iso }}$ values.

\section{Characteristics of Observed GRBs}

The observed GRBs are all somewhat different in their individual behavior, but there are some general themes that can be drawn from the four confirmed detections, which may aid future detection of gamma rays at very high energies. The first obvious similarity is that the four significantly detected GRBs are long GRBs (with $\mathrm{T}_{90}$ of 30 s or above), likely originating from massive star collapse. Figure 1 shows the brightness of the VHE GRBs in 
comparison to the distribution of gamma-ray fluences as measured by Swift-BAT ${ }^{1}$ and the eleven hour X-ray flux measured by Swift-XRT. Firstly, it is clear from both distributions that the VHE GRBs are all relatively bright in the keV energy range, with GRBs 180720B, 190114C and 201216C lying in the top 1\% of all prompt GRB fluences. Similarly, the VHE GRBs are also bright in comparison to the distribution of $\mathrm{X}$-ray afterglow emission, especially the two GRBs observed in the deep afterglow phase (GRBs 180720B and 190829A).
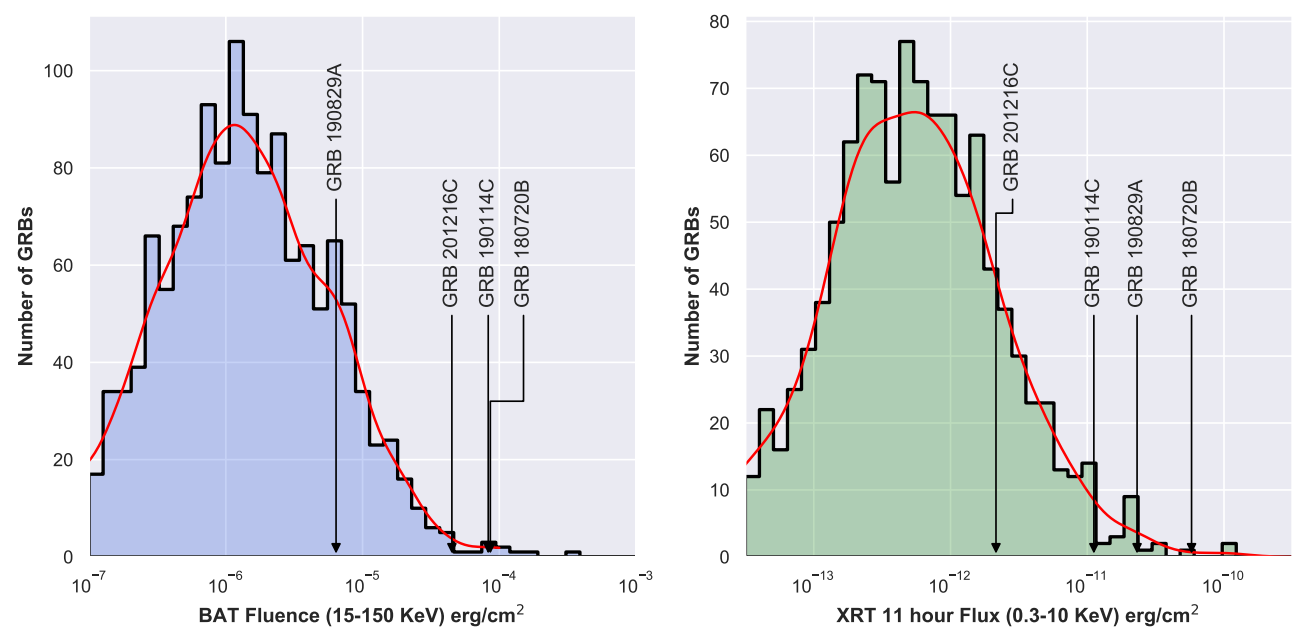

Figure 1. Distributions of the brightness of GRBs observed by Swift in the prompt and deep afterglow (11 h delay) phases. Brightness of the four VHE GRBs is marked on the $x$-axis. The red line displays the probability density of the distributions calculated by kernel density estimation.

The classification of the detected GRBs as both long and bright is likely dictated by instrumental constraints, with only the brightest GRBs being detectable given the relatively limited sensitivity of the current generation of Cherenkov telescopes and shorter events being difficult to detect due to their rapid fall off in brightness [76]. In addition, the brightness of the VHE burst and the correlations between the VHE gamma-ray and X-ray light curves implies that the lower energy gamma-ray and X-ray emissions represent a reasonable proxy for the gamma-ray brightness and could be used as a selection criteria for GRBs to be observed by VHE instruments.

This behavior is borne out in the comparison of X-ray lightcurves ${ }^{2}$ shown in Figure 2 (left), with all four GRBs showing a similar brightness. However, GRB 190829A does shows a significant difference in the light curve behavior from the others, peaking more brightly, rapidly dimming and undergoing a re-brightening phase around $1000 \mathrm{~s}$ after the burst time. When representing the light curves as luminosity in Figure 2 (right), the nature of GRB 190829A as an outlier is even more clear, lying significantly below the other GRBs. The sub-threshold GRB 201015A shows a rather similar behavior to GRB 190829A but without the rebrightening; however, it should be noted that significant gaps are present in the Swift-XRT coverage of this burst (interpolated as a logarithmic decrease in Figure 2).

When combined with the redshift vs. isotropic energy output, ${ }^{3}$ distribution plot shown in Figure 3 it becomes clear that the observed GRBs originate from two rather different distributions and represent different event classes. It has been proposed in literature that the brighter GRBs represent a distinct class of binary driven hypernovae (e.g., [77]), while GRB 190829A represents a low luminosity GRB (e.g., [78]); however, of course, with such a small number of events, such classifications remain speculative. Regardless of the physical processes involved within the GRB, it is clear that the GRB detections made by the current generation of Cherenkov telescopes are rather extreme events, skirting around the edges of the distribution of GRBs so far seen. Therefore, it should be considered that any conclusions drawn from this small event sample may not necessarily be representative of the majority of gamma-ray bursts. However, with increased instrument sensitivity, there is a clearly significant scope to vastly increase the number of VHE gamma-ray bursts detected. 

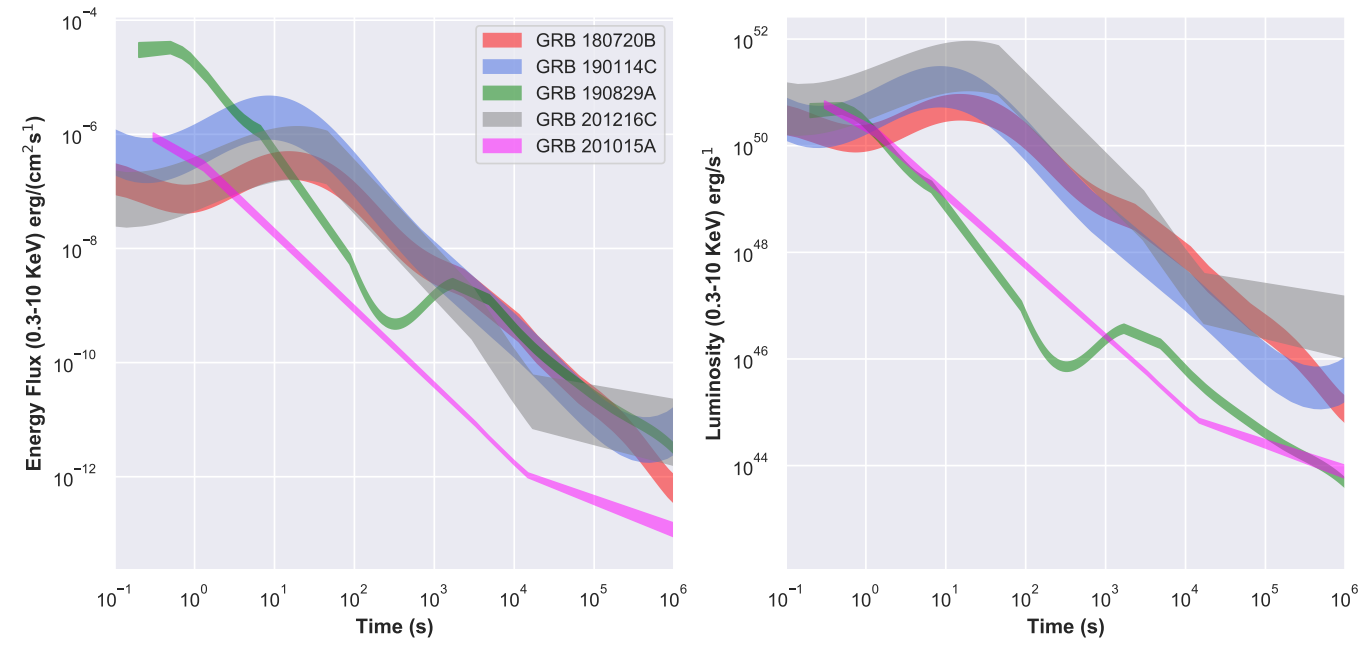

Figure 2. X-ray light curve of the four VHE GRBs shown as flux (left) and luminosity (right) using data from the Swift-BAT and XRT. Curves represent a smooth interpretation of the light curve evolution (using Gaussian processes); the width of the line gives an indication of the spread of data points. GRB 160821B is not shown in this plot because, as a short GRB, it is not directly comparable.

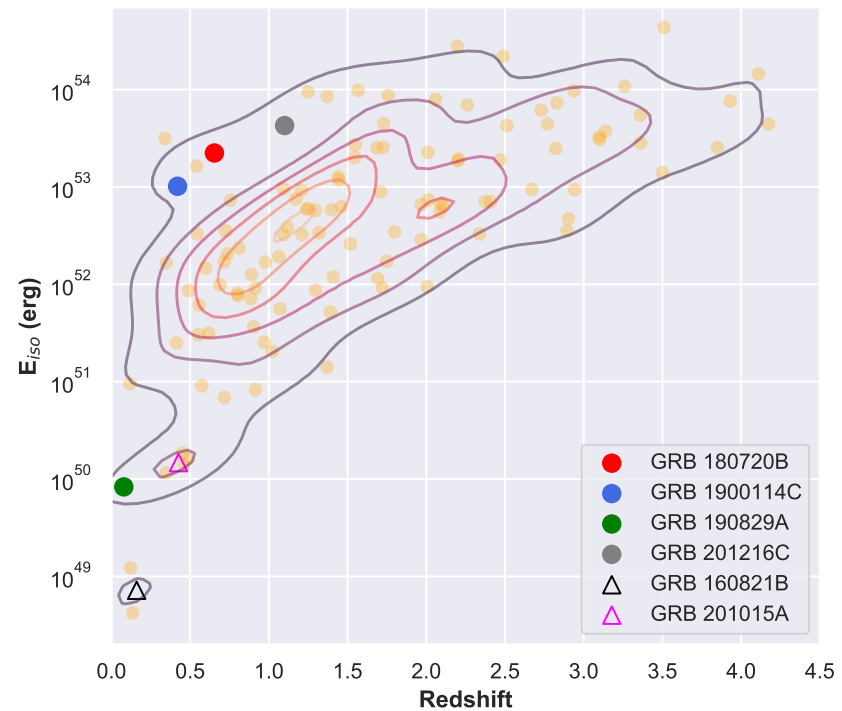

Figure 3. Distribution of the isotropic energy output of the GRBs (in the $50-300 \mathrm{keV}$ range) versus the redshift. VHE GRBs are marked with the colored points (sub threshold GRBs are marked with open points), contours show the probability distribution calculated by kernel density estimation.

Finally, it is clear from Figure 3 that the sub-threshold GRB 160821B stands quite far apart from the main body of GRBs (with known redshift); however, this is to be expected due to its nature as a short GRB.

\section{Conclusions and Prospects}

This paper summarized the status of GRB observations by the IACTs that progressed tremendously in recent years. The instruments and observational strategies by those collaborations were briefly described, as well as the four clear and two more subthreshold GRB detections were explained in detail. The detection of these GRBs has proven the existence of emissions above $100 \mathrm{GeV}$ at a flux level detectable by the relatively small instruments currently available. Although the number the detections is small, we have also tried to characterize the GRBs with VHE gamma-ray emissions in order to catch a glimpse of the hidden distribution behind the detected events. However, the detections made so far 
remain rather extreme bursts, on the edges of the distribution of events. We could not yet draw a clear conclusion if the detections are representing the majority of the GRBs.

Anyway, the detections have improved the observational strategies, and have also given insights into the emission mechanisms. The current generation of ground based gamma-ray telescopes all have active observation programs for gamma-ray bursts, and thus they will continue to regularly make observations of gamma-ray burst events for the years to come, expanding their observation programs to cover the several day timescales over which GRBs have been detected. Even though it is not the main scope of this paper, $\mathrm{wFoV}$ ground-based gamma-ray telescopes also have great potential to make a significant contribution in this field, by a synergy with the IACTs.

Within the decade, the next generation ground based gamma-ray observatory, the Cherenkov Telescope Array (CTA), should be completed [27]. CTA will combine multiple telescope types in both the northern and southern hemispheres to provide a sensitivity increase of an order of magnitude of the current telescope generation in the energy range from tens of $\mathrm{GeV}$ to hundreds of $\mathrm{TeV}$. In particular, the large size (low energy optimised) telescopes are optimized for rapid repointing toward transient sources and should allow GRBs to be observed close to the burst time with significantly increased sensitivity. The large number of large and medium sized telescopes will also allow deep observations of GRBs in the afterglow regime. CTA observations will allow us to significantly increase the number of VHE detections, allowing the analysis of a much more representative sample of GRBs out to much longer timescales, greatly improving our understanding of particle acceleration within GRBs.

Funding: This research was funded by JSPS KAKENHI grant No. JP20KK0067 from MEXT, Japan.

Acknowledgments: We are thankful for the support by H.E.S.S., MAGIC, and VERITAS collaborations. In particular, we would like to acknowledge Lara Nava, Deivid Ribeiro, and Fabian Schüssler, who gave us useful comments and information.

Conflicts of Interest: The authors declare no conflict of interest.

\section{Notes}

Data from https:/ /swift.gsfc.nasa.gov/archive/grb_table/ [accessed on 2 September 2021].

Data from https://www.swift.ac.uk/xrt_products / [accessed on 23 December 2021].

Data from https:/ /heasarc.gsfc.nasa.gov/W3Browse/fermi/fermigbrst.html [accessed on 2 December 2021].

\section{References}

1. Klebesadel, R.W.; Strong, I.B.; Olson, R.A. Observations of Gamma-Ray Bursts of Cosmic Origin. Astrophys. J. Lett. 1973, 182, L85. [CrossRef]

2. BATSE Website. Available online: https://gammaray.msfc.nasa.gov/batse/ (accessed on 31 December 2021).

3. HETE-II Website. Available online: https://space.mit.edu/HETE/ (accessed on 31 December 2021).

4. Meegan, C.A.; Fishman, G.J.; Wilson, R.B.; Paciesas, W.S.; Pendleton, G.N.; Horack, J.M.; Brock, M.N.; Kouveliotou, C. Spatial distribution of $\gamma$-ray bursts observed by BATSE. Nature 1992, 355, 143-145. [CrossRef]

5. Kouveliotou, C.; Meegan, C.A.; Fishman, G.J.; Bhat, N.P.; Briggs, M.S.; Koshut, T.M.; Paciesas, W.S.; Pendleton, G.N. Identification of Two Classes of Gamma-Ray Bursts. Astrophys. J. Lett. 1993, 413, L101. [CrossRef]

6. Band, D.; Matteson, J.; Ford, L.; Schaefer, B.; Palmer, D.; Teegarden, B.; Cline, T.; Briggs, M.; Paciesas, W.; Pendleton, G.; et al. BATSE Observations of Gamma-Ray Burst Spectra. I. Spectral Diversity. Astrophys. J. 1993, 413, 281. [CrossRef]

7. Gehrels, N.; Chincarini, G.; Giommi, P.; Mason, K.O.; Nousek, J.A.; Wells, A.A.; White, N.E.; Barthelmy, S.D.; Burrows, D.N.; Cominsky, L.R.; et al. The Swift Gamma-Ray Burst Mission. Astrophys. J. 2004, 611, 1005-1020. [CrossRef]

8. GCN: The Gamma-ray Coordinates Network. Available online: https://gcn.gsfc.nasa.gov/ (accessed on 31 December 2021).

9. Meegan, C.; Lichti, G.; Bhat, P.N.; Bissaldi, E.; Briggs, M.S.; Connaughton, V.; Diehl, R.; Fishman, G.; Greiner, J.; Hoover, A.S.; et al. The Fermi Gamma-ray Burst Monitor. Astrophys. J. 2009, 702, 791-804. [CrossRef]

10. Atwood, W.B.; Abdo, A.A.; Ackermann, M.; Althouse, W.; Anderson, B.; Axelsson, M.; Baldini, L.; Ballet, J.; Band, D.L.; Barbiellini, G.; et al. The Large Area Telescope on the Fermi Gamma-Ray Space Telescope Mission. Astrophys. J. 2009, 697, 1071-1102. [CrossRef]

11. von Kienlin, A.; Meegan, C.A.; Paciesas, W.S.; Bhat, P.N.; Bissaldi, E.; Briggs, M.S.; Burns, E.; Cleveland, W.H.; Gibby, M.H.; Giles, M.M.; et al. The Fourth Fermi-GBM Gamma-Ray Burst Catalog: A Decade of Data. Astrophys. J. 2020, 893, 46. [CrossRef] 
12. Bromberg, O.; Nakar, E.; Piran, T.; Sari, R. Short versus Long and Collapsars versus Non-collapsars: A Quantitative Classification of Gamma-Ray Bursts. Astrophys. J. 2013, 764, 179. [CrossRef]

13. Ajello, M.; Arimoto, M.; Axelsson, M.; Baldini, L.; Barbiellini, G.; Bastieri, D.; Bellazzini, R.; Bhat, P.N.; Bissaldi, E.; Blandford, R.D.; et al. A Decade of Gamma-Ray Bursts Observed by Fermi-LAT: The Second GRB Catalog. Astrophys. J. 2019, 878, 52. [CrossRef]

14. Franceschini, A.; Rodighiero, G.; Vaccari, M. Extragalactic optical-infrared background radiation, its time evolution and the cosmic photon-photon opacity. Astron. Astrophys. 2008, 487, 837-852. [CrossRef]

15. Wakely, S.P.; Horan, D. TeVCat: An online catalog for Very High Energy Gamma-Ray Astronomy. In Proceedings of the 30th International Cosmic Ray Conference, Merida, Yucatan, Mexico, 3-11 July 2007; Volume 3, pp. 1341-1344.

16. Fioretti, V.; Ribeiro, D.; Humensky, T.B.; Bulgarelli, A.; Maier, G.; Moralejo, A.; Nigro, C. The Cherenkov Telescope Array sensitivity to the transient sky. arXiv 2019, arXiv:1907.08018.

17. Holler, M.; Berge, D.; van Eldik, C.; Lenain, J.P.; Marandon, V.; Murach, T.; de Naurois, M.; Parsons, R.D.; Prokoph, H.; Zaborov, D. Observations of the Crab Nebula with H.E.S.S. Phase II. arXiv 2015, arXiv:1509.02902.

18. Aharonian, F.; Akhperjanian, A.G.; Barres de Almeida, U.; Bazer-Bachi, A.R.; Behera, B.; Benbow, W.; Bernlöhr, K.; Boisson, C.; Bochow, A.; Borrel, V.; et al. HESS observations of $\gamma$-ray bursts in 2003-2007. Astron. Astrophys. 2009, 495, 505-512. [CrossRef]

19. Hoischen, C.; Balzer, A.; Bissaldi, E.; Füßling, M.; Garrigoux, T.; Gottschall, D.; Holler, M.; Mitchell, A.; O’Brien, P.; Parsons, R.; et al. GRB Observations with H.E.S.S. II. arXiv 2017, arXiv:1708.01088.

20. Parsons, R.; Balzer, A.; Fuessling, M.; Hoischen, C.; Holler, M.; Mitchell, A.; Puehlhofer, G.; Rowell, G.; Wagner, S.; Bissaldi, E.; et al. The H.E.S.S. II GRB Observation Program. In Proceedings of the 34th International Cosmic Ray Conference (ICRC2015), Hague, The Netherlands, 30 July-6 August 2015; p. 853.

21. Piel, Q.; Arcaro, C.; Ashkar, H.; Bissaldi, E.; Böttcher, M.; Egbert, K.; Carosi, A.; Hoischen, C.; Holler, M.; O’Brien, P.; et al. Gamma-Ray Burst observation at Very High Energy with H.E.S.S. In Proceedings of the 36th International Cosmic Ray Conference (ICRC-2019) (The Astroparticle Physics Conference), Madison, WI, USA, 24 July-1 August 2019; Volume 36, p. 761. [CrossRef]

22. Aleksić, J.; Ansoldi, S.; Antonelli, L.A.; Antoranz, P.; Babic, A.; Bangale, P.; Barceló, M.; Barrio, J.A.; Becerra González, J.; Bednarek, W.; et al. The major upgrade of the MAGIC telescopes, Part II: A performance study using observations of the Crab Nebula. Astropart. Phys. 2016, 72, 76-94. [CrossRef]

23. Longo, F.; Berti, A.; Bosnjak, Z.; Donini, A.; Fukami, S.; Green, J.G.; Miceli, D.; Moretti, E.; Nava, L.; Noda, K.; et al. Upper limits on the very high energy emission from GRBs observed by MAGIC. In Proceedings of the 37th International Cosmic Ray Conference-PoS(ICRC2021), Berlin, Germany, 12-23 July 2021; Volume 395, p. 820. [CrossRef]

24. Holder, J.; Atkins, R.W.; Badran, H.M.; Blaylock, G.; Bradbury, S.M.; Buckley, J.H.; Byrum, K.L.; Carter-Lewis, D.A.; Celik, O.; Chow, Y.C.K.; et al. The first VERITAS telescope. Astropart. Phys. 2006, 25, 391-401. [CrossRef]

25. Park, N.; VERITAS Collaboration. Performance of the VERITAS experiment. In Proceedings of the 34th International Cosmic Ray Conference (ICRC2015), Hague, The Netherlands, 30 July-6 August 2015; Volume 34, p. 771.

26. Ribeiro, D.; the VERITAS Collaboration. The VERITAS Gamma-Ray Burst Follow-up Program. In AAS/High Energy Astrophysics Division; American Astronomical Society: Washington, DC, USA, 2021.

27. CTA-Consortium. Science with the Cherenkov Telescope Array; World Scientific: Singapore, 2019. [CrossRef]

28. Abeysekara, A.U.; Albert, A.; Alfaro, R.; Alvarez, C.; Álvarez, J.D.; Arceo, R.; Arteaga-Velázquez, J.C.; Solares, H.A.A.; Barber, A.S.; Bautista-Elivar, N.; et al. Observation of the Crab Nebula with the HAWC Gamma-Ray Observatory. Astrophys. J. 2017, 843, 39. [CrossRef]

29. Amenomori, M.; Ayabe, S.; Chen, D.; Cui, S.W.; Danzengluobu; Ding, L.K.; Ding, X.H.; Feng, C.F.; Feng, Z.Y.; Gao, X.Y.; et al. A Northern Sky Survey for Steady Tera-Electron Volt Gamma-Ray Point Sources Using the Tibet Air Shower Array. Astrophys. J. 2005, 633, 1005-1012. [CrossRef]

30. Di Sciascio, G.; LHAASO Collaboration. The LHAASO experiment: From Gamma-Ray Astronomy to Cosmic Rays. Nucl. Part. Phys. Proc. 2016, 279, 166-173. [CrossRef]

31. Albert, J.; Aliu, E.; Anderhub, H.; Antoranz, P.; Armada, A.; Asensio, M.; Baixeras, C.; Barrio, J.A.; Bartelt, M.; Bartko, H.; et al. Flux Upper Limit on Gamma-Ray Emission by GRB 050713a from MAGIC Telescope Observations. Astrophys. J. Lett. 2006, 641, L9-L12. [CrossRef]

32. Albert, J.; Aliu, E.; Anderhub, H.; Antoranz, P.; Armada, A.; Baixeras, C.; Barrio, J.A.; Bartko, H.; Bastieri, D.; Becker, J.; et al MAGIC Upper Limits on the Very High Energy Emission from Gamma-Ray Bursts. Astrophys. J. 2007, 667, 358-366. [CrossRef]

33. Aleksić, J.; Anderhub, H.; Antonelli, L.A.; Antoranz, P.; Backes, M.; Baixeras, C.; Balestra, S.; Barrio, J.A.; Bastieri, D.; Becerra González, J.; et al. MAGIC observation of the GRB 080430 afterglow. Astron. Astrophys. 2010, 517, A5. [CrossRef]

34. Aleksić, J.; Ansoldi, S.; Antonelli, L.A.; Antoranz, P.; Babic, A.; de Almeida, U.B.; Barrio, J.A.; González, J.B.; Bednarek, W.; Berger, K.; et al. MAGIC upper limits on the GRB 090102 afterglow. Mon. Not. R. Astron. Soc. 2013, 437, 3103-3111. [CrossRef]

35. Aliu, E.; Aune, T.; Barnacka, A.; Beilicke, M.; Benbow, W.; Berger, K.; Biteau, J.; Buckley, J.H.; Bugaev, V.; Byrum, K.; et al. Constraints on Very High Energy Emission from GRB 130427A. Astrophys. J. Lett. 2014, 795, L3. [CrossRef]

36. Abramowski, A.; Aharonian, A.; Benkhali, F.; Akhperjanian, F.A.; Angüner, A.G.; Anton, E.; Balenderan, G.; Balzer, S.; Barnacka, A.; Becherini, A.; et al. Search for TeV Gamma-ray Emission from GRB 100621A, an extremely bright GRB in X-rays, with H.E.S.S. Astron. Astrophys. 2014, 565, A16. [CrossRef]

37. Siegel, M.H. GCN22973-GRB 180720B: Swift detection of a burst. GCN Circ. 2018, 22973, 96-100.

38. Roberts, O.J. GCN22981-GRB 180720B: Fermi-GBM observation. GCN Circ. 2018, 22981, 96. 
39. Bissaldi, E. GCN22980-GRB 180720B: Fermi-LAT detection. GCN Circ. 2018, 22980, 96-100.

40. Malesani, D. GCN22996-VLT/X-shooter redshift. GCN Circ. 2018, $22996,97$.

41. Abdalla, H.; Adam, R.; Aharonian, F.; Ait Benkhali, F.; Angüner, E.O.; Arakawa, M.; Arcaro, C.; Armand, C.; Ashkar, H.; Backes, M.; et al. A very-high-energy component deep in the $\gamma$-ray burst afterglow. Nature 2019, 575, 464-467. [CrossRef]

42. Hamburg, R.; Veres, P.; Meegan, C.; Burns, E.; Connaughton, V.; Goldstein, A.; Kocevski, D.; Roberts, O.J. GRB 190114C: Fermi GBM detection. GRB Coord. Netw. 2019, 23707, 1.

43. Krimm, H.A.; Barthelmy, S.D.; Cummings, J.R.; Gropp, J.D.; Lien, A.Y.; Markwardt, C.B.; Palmer, D.M.; Sakamoto, T.; Stamatikos, M.; Ukwatta, T.N. GRB 190114C: Swift-BAT refined analysis. GRB Coord. Netw. 2019, 23724, 1.

44. Selsing, J.; Fynbo, J.P.U.; Heintz, K.E.; Watson, D. GRB 190114C: NOT optical counterpart and redshift. GRB Coord. Netw. 2019, 23695, 1.

45. Castro-Tirado, A.J.; Hu, Y.; Fernandez-Garcia, E.; Valeev, A.; Sokolov, V.; Guziy, S.; Oates, S.; Jeong, S.; Pandey, S.B.; Carrasco, I.; et al. GRB 190114C: refined redshift by the 10.4m GTC. GRB Coord. Netw. 2019, 23708, 1.

46. Mirzoyan, R.; Noda, K.; Moretti, E.; Berti, A.; Nigro, C.; Hoang, J.; Micanovic, S.; Takahashi, M.; Chai, Y.; Moralejo, A.; et al. MAGIC detects the GRB 190114C in the TeV energy domain. GRB Coord. Netw. 2019, 23701, 1.

47. Mirzoyan, R. First time detection of a GRB at sub-TeV energies; MAGIC detects the GRB 190114C. Astron. Telegr. 2019, 12390, 1 .

48. Ajello, M.; Arimoto, M.; Axelsson, M.; Baldini, L.; Barbiellini, G.; Bastieri, D.; Bellazzini, R.; Berretta, A.; Bissaldi, E.; Blandford, R.D.; et al. Fermi and Swift Observations of GRB 190114C: Tracing the Evolution of High-energy Emission from Prompt to Afterglow. Astrophys. J. 2020, 890, 9. [CrossRef]

49. MAGIC Collaboration.; Acciari, V.A.; Ansoldi, S.; Antonelli, L.A.; Arbet Engels, A.; Baack, D.; Babić, A.; Banerjee, B.; Barres de Almeida, U.; Barrio, J.A.; et al. Teraelectronvolt emission from the $\gamma$-ray burst GRB 190114C. Nature 2019, 575, 455-458. [CrossRef]

50. MAGIC Collaboration.; Acciari, V.A.; Ansoldi, S.; Antonelli, L.A.; Engels, A.A.; Baack, D.; Babić, A.; Banerjee, B.; Barres de Almeida, U.; Barrio, J.A.; et al. Observation of inverse Compton emission from a long $\gamma$-ray burst. Nature 2019, 575, 459-463. [CrossRef]

51. Fermi GBM Team. GRB 190829A: Fermi GBM Final Real-time Localization. GRB Coord. Netw. 2019, 25551. Available online: https:/ / ui.adsabs.harvard.edu/abs/2019GCN.25551...1F/abstract (accessed on 31 December 2021).

52. Dichiara, S.; Bernardini, M.G.; Burrows, D.N.; D'Avanzo, P.; Gronwall, C.; Gropp, J.D.; Kennea, J.A.; Klingler, N.J.; Krimm, H.A.; Kuin, N.P.M.; et al. GRB 190829A: Swift detection of a burst consistent with a galaxy at z =0.08. GRB Coord. Netw. $2019,25552$.

53. Osborne, J.P.; Page, K.L.; Capalbi, M.; Perri, M.; D’Elia, V.; Burrows, D.N.; Gropp, J.D.; Tohuvavohu, A.; Beardmore, A.P.; Dichiara, S.; et al. GRB 190829A: Swift-XRT refined Analysis. GRB Coord. Netw. 2019, 25568.

54. Hu, Y.D.; Castro-Tirado, A.J.; Kumar, A.; Gupta, R.; Valeev, A.F.; Pandey, S.B.; Kann, D.A.; Castellón, A.; Agudo, I.; Aryan, A.; et al. $10.4 \mathrm{~m}$ GTC observations of the nearby VHE-detected GRB 190829A/SN 2019oyw. Astron. Astrophys. 2021, 646, A50. [CrossRef]

55. H. E. S. S. Collaboration.; Abdalla, H.; Aharonian, F.; Ait Benkhali, F.; Angüner, E.O.; Arcaro, C.; Armand, C.; Armstrong, T.; Ashkar, H.; Backes, M.; et al. Revealing X-ray and gamma ray temporal and spectral similarities in the GRB 190829A afterglow. Science 2021, 372, 1081-1085. [CrossRef]

56. Beardmore, A.P.; Gropp, J.D.; Kennea, J.A.; Klingler, N.J.; Laha, S.; Lien, A.Y.; Moss, M.J.; Page, K.L.; Palmer, D.M.; Tohuvavohu, A.; et al. GRB 201216C: Swift detection of a burst. GRB Coord. Netw. 2020, 29061, 1.

57. Ukwatta, T.N.; Barthelmy, S.D.; Beardmore, A.P.; Krimm, H.A.; Laha, S.; Lien, A.Y.; Markwardt, C.B.; Palmer, D.M.; Sakamoto, T.; Stamatikos, M. GRB 201216C: Swift-BAT refined analysis. GRB Coord. Netw. 2020, 29080, 1.

58. Malacaria, C.; Veres, P.; Meegan, C.; Bissaldi, E.; Fermi GBM Team. GRB 201216C: Fermi GBM detection. GRB Coord. Netw. 2020, $29073,1$.

59. Vielfaure, J.B.; Izzo, L.; Xu, D.; Vergani, S.D.; Malesani, D.B.; de Ugarte Postigo, A.; D’Elia, V.; Fynbo, J.P.U.; Kann, D.A.; Levan, A.J.; et al. GRB 201216C: VLT X-shooter spectroscopy and potential high redshift of a VHE-emitting GRB. GRB Coord. Netw. 2020, $29077,1$.

60. Blanch, O.; Longo, F.; Berti, A.; Fukami, S.; Suda, Y.; Loporchio, S.; Micanovic, S.; Green, J.G.; Pinter, V.; Takahashi, M.; et al. GRB 201216C: MAGIC detection in very high energy gamma rays. GRB Coord. Netw. 2020, $29075,1$.

61. Fukami, S.; Berti, A.; Loporchio, S.; Suda, Y.; Nava, L.; Noda, K.; Bosnjak, Z.; Asano, K.; Longo, F.; Acciari, V.A.; et al. Veryhigh-energy gamma-ray emission from GRB 201216C detected by MAGIC. In Proceedings of the 37th International Cosmic Ray Conference-PoS(ICRC2021), Berlin, Germany, 15-22 July 2021; Volume 395, p. 788. [CrossRef]

62. Siegel, M.H.; Barthelmy, S.D.; Burrows, D.N.; Lien, A.Y.; Marshall, F.E.; Palmer, D.M.; Sbarufatti, B. GRB 160821B: Swift detection of a short burst. GRB Coord. Netw. 2016, 19833,1.

63. Stanbro, M.; Meegan, C. GRB 160821B: Fermi GBM Detection. GRB Coord. Netw. 2016, 19843, 1.

64. Xu, D.; Malesani, D.; de Ugarte Postigo, A.; Gafton, E.; Rivero Losada, I. GRB 160821B: NOT optical afterglow candidate. GRB Coord. Netw. 2016, 19834,1.

65. Acciari, V.A.; Ansoldi, S.; Antonelli, L.A.; Arbet Engels, A.; Asano, K.; Baack, D.; Babić, A.; Baquero, A.; Barres de Almeida, U.; Barrio, J.A.; et al. MAGIC Observations of the Nearby Short Gamma-Ray Burst GRB 160821B. ApJ 2021, 908, 90. [CrossRef]

66. Lamb, G.P.; Tanvir, N.R.; Levan, A.J.; de Ugarte Postigo, A.; Kawaguchi, K.; Corsi, A.; Evans, P.A.; Gompertz, B.; Malesani, D.B.; Page, K.L.; et al. Short GRB 160821B: A Reverse Shock, a Refreshed Shock, and a Well-sampled Kilonova. Astrophys. J. 2019, 883, 48. [CrossRef] 
67. Troja, E.; Castro-Tirado, A.J.; Becerra González, J.; Hu, Y.; Ryan, G.S.; Cenko, S.B.; Ricci, R.; Novara, G.; Sánchez-Rámirez, R.; Acosta-Pulido, J.A.; et al. The afterglow and kilonova of the short GRB 160821B. Mon. Not. R. Astron. Soc. 2019, 489, 2104-2116. [CrossRef]

68. D’Elia, V.; Ambrosi, E.; Barthelmy, S.D.; D’Ai, A.; Gropp, J.D.; Klingler, N.J.; Lien, A.Y.; Palmer, D.M.; Sbarufatti, B.; Siegel, M.H.; et al. GRB 201015A: Swift detection of a burst. GRB Coord. Netw. 2020, 28632, 1

69. Markwardt, C.B.; Barthelmy, S.D.; Cummings, J.R.; D’Elia, V.; Krimm, H.A.; Laha, S.; Lien, A.Y.; Palmer, D.M.; Sakamoto, T.; Stamatikos, M.; et al. GRB 201015A: Swift-BAT refined analysis (a soft short pulse with a tail emission). GRB Coord. Netw. 2020, $28658,1$.

70. de Ugarte Postigo, A.; Kann, D.A.; Blazek, M.; Agui Fernandez, J.F.; Thoene, C.; Gomez Velarde, G. GRB 201015A: Redshift from GTC/OSIRIS. GRB Coord. Netw. 2020, 28649, 1.

71. Izzo, L.; Malesani, D.B.; Zhu, Z.P.; Xu, D.; de Ugarte Postigo, A.; Pursimo, T. GRB 201015A: Redshift confirmation. GRB Coord. Netw. 2020, 28661, 1 .

72. Minaev, P.; Pozanenko, A. GRB 201015A: Classification as long GRB. GRB Coord. Netw. 2020, $28668,1$.

73. Pozanenko, A.; Belkin, S.; Volnova, A.; Moskvitin, A.; Burhonov, O.; Kim, V.; Krugov, M.; Rumyantsev, V.; Klunko, E.; Inasaridze, R.Y.; et al. GRB 201015A: Optical observations and supernova identification. GRB Coord. Netw. 2020, $29033,1$.

74. Rossi, A.; Benetti, S.; Palazzi, E.; D’Avanzo, P.; D’Elia, V.; De Pasquale, M.; CIBO Collaboration. GRB 201015A: Evidence of supernova in LBT spectra. GRB Coord. Netw. 2021, 29306, 1.

75. Blanch, O.; Gaug, M.; Noda, K.; Berti, A.; Moretti, E.; Miceli, D.; Gliwny, P.; Ubach, S.; Schleicher, B.; Cerruti, M.; et al. MAGIC observations of GRB 201015A: Hint of very high energy gamma-ray signal. GRB Coord. Netw. 2020, $28659,1$.

76. Kagawa, Y.; Yonetoku, D.; Sawano, T.; Arimoto, M.; Kisaka, S.; Yamazaki, R. Exponentially Decaying Extended Emissions Following Short Gamma-Ray Bursts with a Possible Luminosity-E-folding Time Correlation. Astrophys. J. 2019, $877,147$. [CrossRef]

77. Rueda, J.A.; Ruffini, R.; Wang, Y. Induced Gravitational Collapse, Binary-Driven Hypernovae, Long Gramma-ray Bursts and Their Connection with Short Gamma-ray Bursts. Universe 2019, 5, 110. [CrossRef]

78. Liang, E.; Zhang, B.; Virgili, F.; Dai, Z.G. Low-Luminosity Gamma-Ray Bursts as a Unique Population: Luminosity Function, Local Rate, and Beaming Factor. Astrophys. J. 2007, 662, 1111-1118. [CrossRef] 